\title{
Diverticulopexia no tratamento do divertículo de Zenker
}

\section{Diverticulopexy for the treatment of Zenker's diverticulum}

\author{
Renato Micelli Lupinacci ${ }^{1}$; Antonio Carlos Pereira Lima²; Renato Arioni lupinacci ${ }^{3}$
}

R E S U M O

\begin{abstract}
O divertículo de Zenker é um pseudodivertículo que se origina de um defeito muscular na parede posterior da faringe, na área de transição entre o músculo constritor inferior da faringe e o músculo cricofaringeo. Apesar do avanço das técnicas endoscópicas, o tratamento cirúrgico persiste como o tratamento padrão. Duas técnicas são possíveis: diverticulectomia (ressecção do divertículo) e a diverticulopexia. As vantagens da diverticulopexia estão ligadas à ausência de anastomose esofágica e suas possíveis complicações: fistulas cervicais, mediastinite, estenose esofágica e infecção de ferida. Em ambas as técnicas a secção das fibras musculares do músculo cricofaringeo (ou, esfíncter superior do esôfago) é fundamental. O objetivo do presente artigo é descrever em detalhes a técnica de diverticulopexia junto ao ligamento pré-vertebral associada à miotomia do músculo cricofaríngeo.
\end{abstract}

Descritores: Divertículo. Divertículo esofágico. Divertículo de Zenker. Terapêutica. Procedimentos cirúrgicos operatórios.

\section{INTRODUÇÃO}

$\mathrm{O}$ divertículo de Zenker é um pseudodivertículo, ou divertículo não verdadeiro, que se origina através de defeito muscular na parede posterior da faringe, na área de transição entre o músculo constritor inferior da faringe e o músculo cricofaringeo. Esta zona de fragilidade muscular é também conhecida como triângulo de Killian. Dois tratamentos conceitualmente distintos são propostos para os pacientes portadores de divertículo de Zenker sintomático: cirúrgico (diverticulectomia ou diverticulopexia) ou endoscópico (através de endoscópio rígido ou flexível).

O objetivo deste artigo é descrever em detalhes a técnica de diverticulopexia junto ao ligamento pré-vertebral associada à miotomia do músculo cricofaringeo.

\section{Técnica operatória}

Nós preferimos realizar este procedimento sob anestesia geral, que permite melhor controle da via aérea e monitorização hemodinâmica intraoperatória, entretanto, este-pode ser realizado através de anestesia local.

Após indução anestésica e intubação orotraqueal, o paciente é posicionado para melhor exposição do esôfago cervical. A cânula de intubação é colocada à direita da boca, a cabeça é colocada sobre um coxim e retornada para a direita, outro pequeno coxim é colocado sob os ombros.

A incisão cutânea, iniciada a aproximadamente $3 \mathrm{~cm}$ acima da clavícula e com seis a oito centímetros de comprimento, é realizada ao longo da borda anterior do músculo esternocleidomastoide. O músculo esternocleidomastoide e a bainha carotídea são afastados lateralmente. Nós sempre dividimos o músculo omo-hioideo na tentativa de buscar uma melhor exposição do esôfago cervical. A veia tireoidiana média e a artéria tireoidiana inferior são ligadas.

Uma dissecção cuidadosa é realizada para liberar o esôfago posterior e a faringe do espaço pré-vertebral. Neste momento é importante que a dissecção se dirija diretamente aos planos posteriores e ao espaço pré-vertebral, evitando dissecção do plano anterior e possível lesão do nervo laríngeo recorrente (Figura 1).

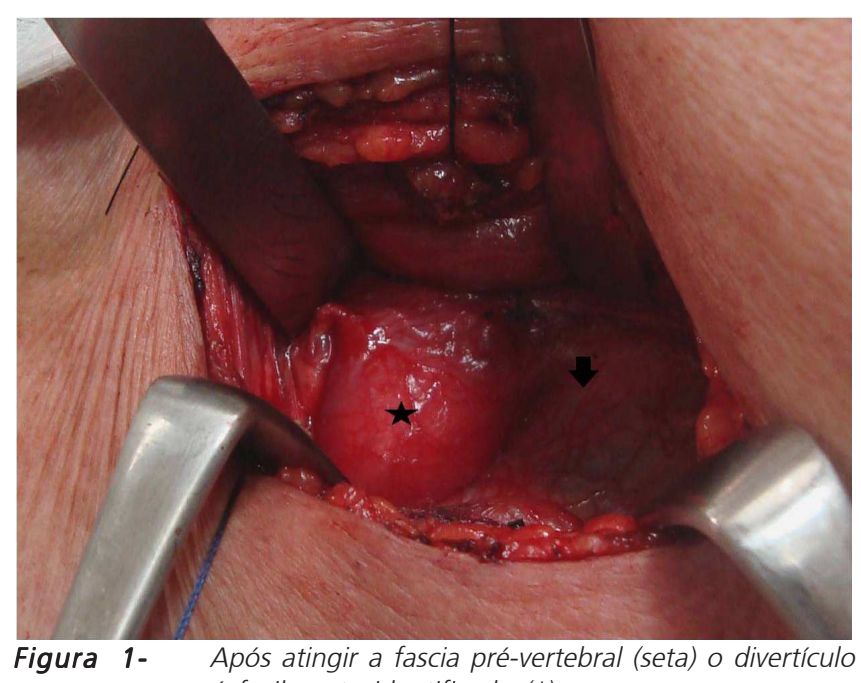
é facilmente identificado (*).

Trabalho realizado no Departamento de Cirurgia; Hospital do Servidor Público Estadual.

1. Assistant Spécialiste, Service de Chirurgie Générale, Viscérale et Endocrinienne - Hôpital Pitié Salpétrière, Paris - FRANCE ; 2 . Médico do Serviço de Cirurgia Geral do Hospital do Servidor Público Estadual-São Paulo; 3. Cirurgião do Serviço de Cirurgia Geral do Hospital do Servidor Público Estadua I-São Paulo. 
Uma vez identificado o divertículo, este é liberado completamente das estruturas adjacentes através de dissecção romba ou com eletrocautério. Nós preferimos começar esta dissecção do esôfago superior em direção à faringe, tracionando o divertículo gradualmente, liberando-o por completo até atingirmos a sua origem na junção dos músculos constritor inferior da faringe e cricofaríngeo. É fundamental a dissecção completa do divertículo e a exposição do seu colo livre de qualquer fibra muscular, desta maneira ele será facilmente mobilizado em direção cranial no momento da sua fixação ao espaço pré-vertebral permitindo a obstrução completa da sua luz.

Uma pinça tipo Kelly ou Mixter é utilizada para a dissecção cuidadosa do músculo cricofaríngeo, separando-o da mucosa e submucosa do esôfago proximal. Realizamos, então, uma miotomia do músculo cricofaríngeo de $3 \mathrm{~cm}$ em direção ao esôfago distalmente. Uma miotomia completa é essencial, pois a descoordenação do relaxamento do músculo cricofaríngeo é, provavelmente, o principal fator patofisiológico ligado ao aparecimento do divertículo (Figura 2).

A fascia pré-vertebral é dissecada a fim de criar espaço suficiente para que o divertículo possa "deitar-se" quando tracionado cranialmente. Nós começamos a fixação por três pontos de prolene 3-0 junto ao ligamento prévertebral (atenção deve ser tomada para evitar o disco prévertebral e a cortical óssea para não causar espondilodiscite ou osteíte), e depois, um a um, através do tecido peridiverticular (Figuras 3 e 4). Finalmente, os nós são amarrados com o objetivo de suspender a totalidade do divertículo, ocluir o colo diverticular e acomodá-lo corretamente sobre a fáscia. Dois outros pontos são acrescentados no intuito de fixar a borda lateral esquerda do divertículo ao ligamento pré-vertebral (Figura 5).

Após a revisão cuidadosa da hemostasia, o músculo omo-hioideo é reaproximado através de ponto em " $U$ " de fio absorvível 2-0. O plano do músculo platisma é aproximado com pontos separados de polyglactin 910 (4-0). A sutura cutânea é realizada com postos contínuos intradérmicos de poliglecaprone 25 (4-0). Embora não seja necessário, nós frequentemente deixamos um dreno de Penrose junto ao espaço pré-vertebral, próximo ao esôfago, durante dois dias.

Dieta líquida é iniciada no primeiro dia pós-operatório, a liberação para alimentos sólidos ocorre após duas semanas. Apesar de não realizarmos controle radiológico pós-operatório rotineiro, este pode ser solicitado ao primeiro ou segundo dia pós-operatório para certificar-se que o divertículo não é preenchido durante a deglutição (Figura $6)$.

\section{DISCUSSÃO}

Ambas as técnicas rotineiras no tratamento do divertículo de Zenker: cirúrgicas ou endoscópicas, melho- ram a disfagia em até $90 \%$ dos pacientes'. Em relação à remissão completa dos sintomas, os resultados também parecem ser comparáveis, muito embora os dados publi-

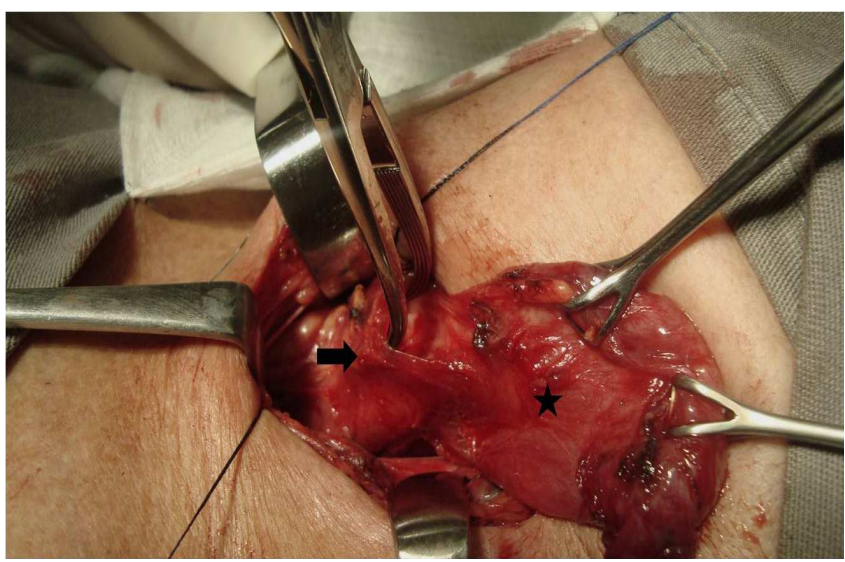

Figura 2 - $\quad$ Realização da miotomia. O divertículo (*) é completamente isolado. Iniciamos a miotomia $(3 \mathrm{~cm})$ do músculo cricofaringeo (seta) a partir do colo diverticular em direção ao esôfago proximal.

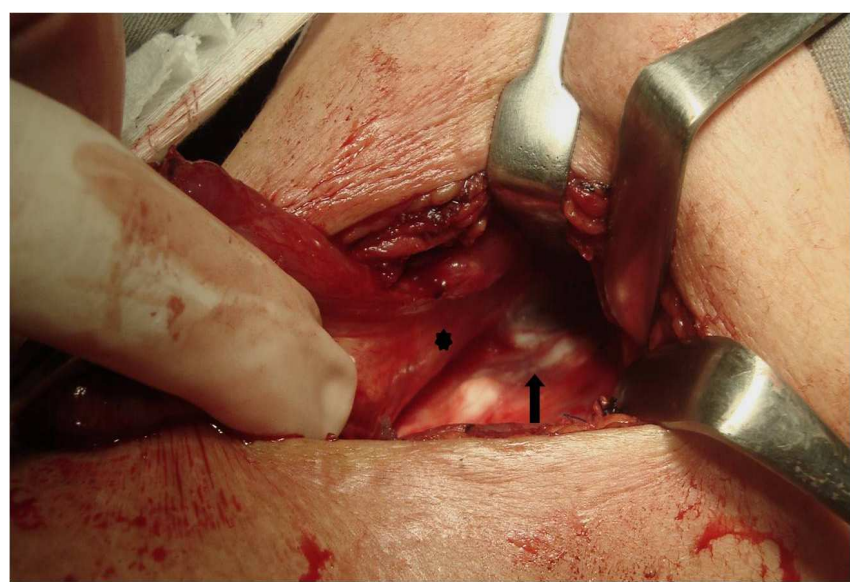

Figura 3 - Espaço pré-vertebral. (*) O esôfago é tracionado lateralmente. A seta indica o ligamento vertebral anterior.

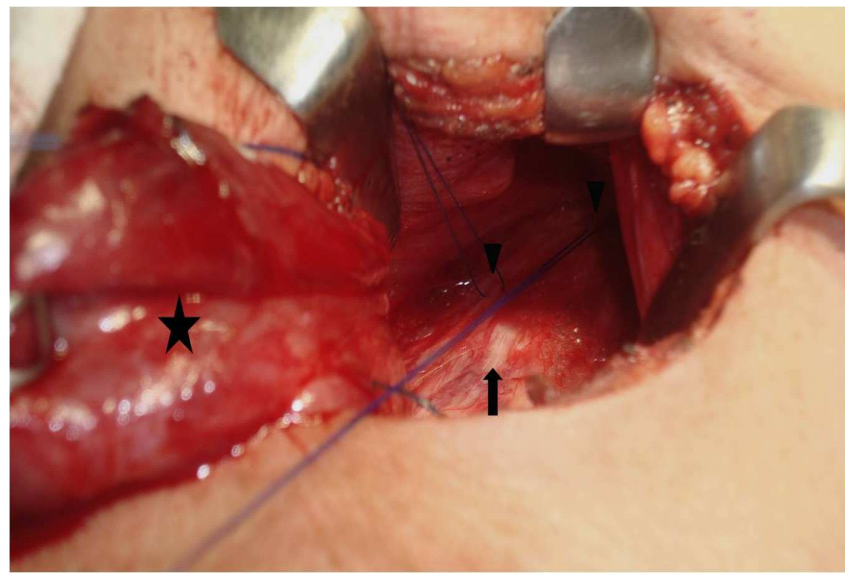

Figura 4 - Fio (pontas de seta) são passados no ligamento prévertebral (seta). O divertículo (*) deve ser completamente suspenso através de sutura bem proximal junto ao ligamento pré-vertebral. 


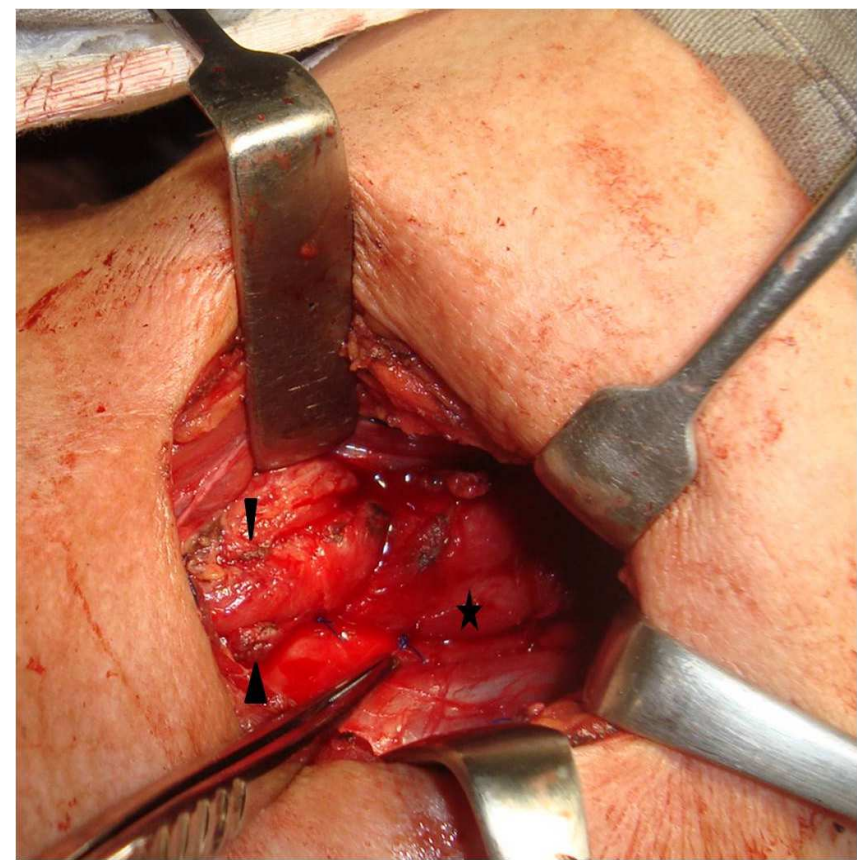

Figura 5 - $\quad$ Aspecto final. (*) Divertículo. As pontas de seta indicam as bordas seccionadas do músculo cricofaríngeo.

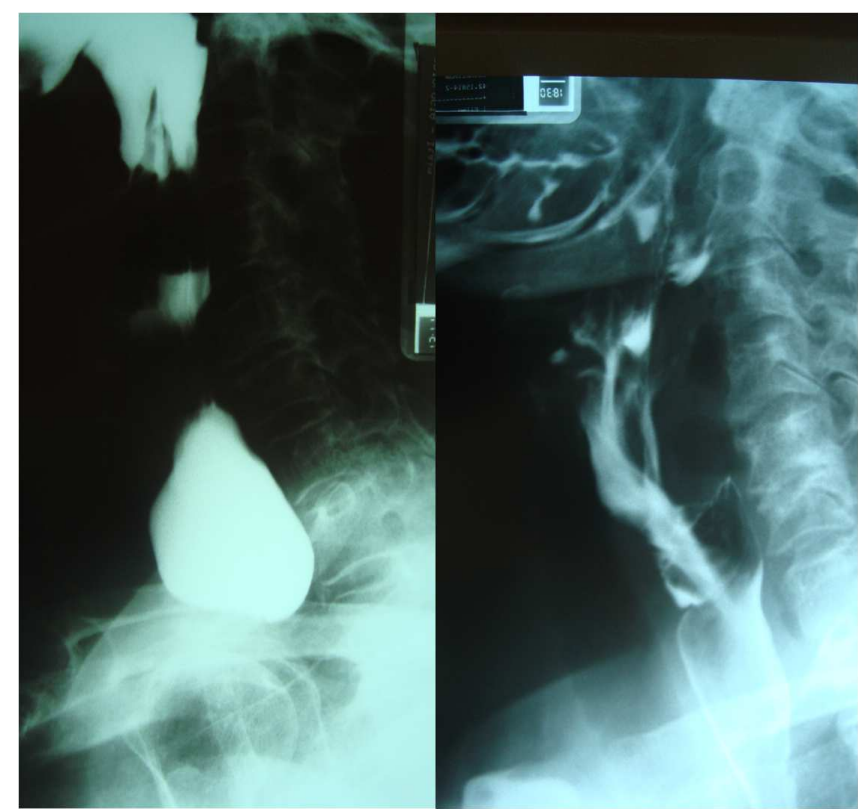

Figura 6 - EED. A) Pré-operatório; B) Primeiro dia pós-operatório. cados sejam pouco elucidativos, posto que escores de disfagia são raramente utilizados e a maioria dos trabalhos apresentam resultados retrospectivos. Outra limitação importante acontece pela variação no tempo de seguimento, apresentando os estudos endoscópicos os menores tempos de seguimento ${ }^{1,2}$

A diverticulostomia (endoscópica) é segura, rápida e eficiente para a maioria dos pacientes portadores de divertículos de Zenker de tamanho médio, porém o tratamento cirúrgico oferece melhores resultados, em longo prazo, como primeiro tratamento, apresentando resultados confiáveis e raramente necessitam de um segundo procedimento. Pequenos divertículos podem representar uma contraindicação formal aos tratamentos endoscópicos, pois um septo excessivamente pequeno não permite uma divisão completa das fibras musculares esfincterianas ${ }^{2,3}$.

A quase totalidade dos autores concorda que um tempo fundamental no tratamento cirúrgico do divertículo de Zenker reside na secção das fibras musculares do músculo cricofaringeo (ou, esfíncter superior do esôfago). Embora pareça evidente atualmente, este conceito é uma aquisição relativamente recente, baseada empiricamente nos achados de níveis significativamente menores de complicações (fístulas) e recidivas quando associa-se a miotomia à simples diverticulectomia 4 .

As vantagens da diverticulopexia estão ligadas à ausência de anastomose esofágica e suas possíveis complicações: fistulas cervicais, mediastinite, estenose esofágica e infecção de ferida, o que permite uma recuperação rápida com aceitação de dieta oral satisfatória ${ }^{2,3,5,6}$. O risco de cancer epidermoide de esôfago não deve ser considerado uma contraindicação às técnicas endoscópicas e, tão pouco, à diverticulopexia, pois, além de uma ocorrência extremamente rara, está associado à divertículos de longa $\operatorname{data}^{3,7,8}$.

Embora alguns autores não considerem a diverticulopexia uma alternativa para divertículos volumosos $(>5 \mathrm{~cm})$, nós acreditamos que através de completa mobilização do divertículo, exposição do colo diverticular livre de fibras musculares, miotomia longa do músculo cricofaringeo e ampla dissecção do espaço pré-vertebral permitindo que o divertículo seja confortavelmente suspenso propiciando um resultado funcional satisfatório e com menos complicações.

\section{A B $S$ S T R A $A$}

Zenker's diverticulum is a pseudodiverticulum through a muscular defect in the posterior pharyngeal wall at the area between the inferior pharyngeal constrictor muscles of the pharynx and the cricopharyngeus muscle. Although endoscopic techniques have made significant progress, the surgical treatment remains the gold standard. There are two main techniques: diverticulectomy (resection of the diverticulum) and diverticulopexy. The main advantages of diverticulopexy are mostly linked to the absence of an esophageal anatomosis and its possible complications: cervical fistulae, mediastinitis, esophageal stenosis and wound infection, which allows a rapid recover with satisfactory oral intake. The purpose of this article is to describe in details the technique for diverticulopexy to the prevertebral ligament in association with a miotomy of the cricopharyngeus muscle.

Key words: Diverticulum. Diverticulum, esophageal. Zenker diverticulum. Therapeutics. Surgical procedures, operative. 


\section{REFERENCIAS}

1. Smith SR, Genden EM, Urken ML. Endoscopic stapling technique for the treatment of Zenker diverticulum vs standard open-neck technique: a direct comparison and charge analysis. Arch Otolaryngol Head Neck Surg. 2002;128(2):141-4.

2. Gutschow CA, Hamoir M, Rombaux P, Otte JB, Goncette L, Collard JM. Management of pharyngoesophageal (Zenker's) diverticulum: which technique? Ann Thorac Surg. 2002;74(5):1677-82; discussion 1682-3.

3. Andreollo NA, Soares Júnior C, Coelho Neto JS, Lopes LR, Brandalise NA, Leonardi LS. Tratamento cirúrgico do divertículo de Zenker. Rev Col Bras Cir. 1998;25(1):9-14

4. Payne WS. The treatment of pahryngoesophageal diverticulum: the simple and the complex. Hepatogastroenterology. 1992;39(2):109-14.

5. Sideris $L$, Chen LQ, Ferraro $P$, Duranceau AC. The treatment of Zenker's diverticula: a review. Semin Thorac Cardiovasc Surg. 1999;11(4):337-51.

6. Hauters $P$, Segol $P$, Leroux $Y$, gignoux $M$. Role of cricopharyngeal myotomy associated with diverticuloplexy in the treatment od Zenker's diverticulum (15 years' experience). Ann Chir. 1988;42(10):726-30
7. Aquino JLB, Said MM, Bordalo R. Carcinoma epidermoide "in situ" em divertículo de Zenker. Rev Col Bras Cir. 2005;32(2):106-7.

8. Bowdler DA, Stell PM. Carcinoma arising in posterior pharyngeal pulsion diverticulum (Zenker's diverticulum). Br J Surg. 1987;74(7):561-3.

Recebido em 10/01/2012

Aceito para publicação em 05/02/2012

Conflito de interesse: nenhum

Fonte de financiamento: nenhuma

\section{Como citar este artigo:}

Lupinacci RM, Lima ACP, Lupinacci RA. Diverticulopexia no tratamento do divertículo de Zenker. Rev Col Bras Cir. [periódico na Internet] 2013;40(1). Disponível em URL: http://www.scielo.br/rcbc

\section{Endereço para correspondência:}

Renato Micelli Lupinacci

E-mail: rmlupinacci@gmail.com 